\title{
An Efficient on Demand Load Balancing Routing Protocol for MANETs (EDLBP)
}

\author{
R. K. Singh \\ Department of Electronics \& \\ Communication \\ Uttarakhand Technical \\ University, Dehradun
}

\author{
Jayant Vats ${ }^{1}$, Sachin \\ Khurana $^{1}$ \\ ${ }^{1}$ Department of management \\ Studies and Computer \\ Applications \\ Amritsar College of Engineering \\ \& Technology
}

\author{
Vishal Sharma \\ Department of Computer \\ Science \& Engineering \\ Amritsar College of \\ Engineering \& Technology
}

\begin{abstract}
MANETs are networks capable of communicating in a set of small, low cost, low power sensing devices. A wireless sensor networks is totally based on the limiting factor i.e. energy consumption. A wireless sensor network consists of large number of sensor nodes distributed or scattered in particular network region. MANETs consist of node that is highly mobile, so in particular the range of the nodes is very important. Each device in a MANETs is free to move independently in any direction, and will therefore change its links to other devices frequently. The energy and the bandwidth of such path are of major concern. The lifetime of the network depends upon these parameters. The load balancing is the issue of selection of such optimized paths that are economical, efficient as well as do not allow the network structure to break up in adverse condition. In this paper, we are working on the concept of performance optimization of load balancing routing protocol for MANETs. , AODV protocol and improved it by using NS-2 simulator. The formula and techniques given in this paper can be used to solve the problem regarding the transmission.
\end{abstract}

\section{Keywords}

MANETs, AODV, Load Balancing

\section{INTRODUCTION}

The network model has different nature as the protocol for this is to be designed in the manner that the infrastructure of the cellular network should be considered [1]. The main challenges regarding this are the mobility of nodes and the scalability of the network [2]. Also the performance measurements in terms of energy and the bandwidth efficiency are unknown [6]. In the wireless ad hoc networks all the nodes transmit data towards the source by passing them through the intermediate relaying nodes. The main concept regarding these relaying nodes is that these nodes require processing energy for their transmission success, thus more the number of nodes more is the requirement of the processing energy [6][3]. Whenever single link situation is considered, there is a tradeoff between the energy efficiency and the bandwidth efficiency for wireless multi hop ad hoc networks [1]. The issue for the multi hop network is regarding the scheduling at the link layer and relaying of data at the network layer. It should always be clear that the data in the network is in the form of packets [6]. The whole network considered to be shared medium between all the nodes that can be source, destination or the relaying nodes. It has also been shown that the per node throughput capacity of ad hoc networks with nodes $n$ decreases with $n$ as $\Theta(1 / n \log n)^{1 / 2}$ [3]. The issue regarding this has been shown as the general capacity cost function of channel capacity for arbitrary input alphabets was studied on single link [4]. In the related work the bits per joule capacity of the network is assumed [3]. The tradeoff between energy and the bandwidth has been analyzed under various assumptions on the channel condition and the interference under a linear equidistant relaying network model without considering the energy consumption at the receiver end [5][6][7]. Also the receiver consumption can be improved by using the cross layer design including the effects of the power amplifier used at the transmitter end [8]. The transport efficiency of an ad hoc network was defined considering the transmitter energy and the receiver's processing energy [9] [10]. Thus the energy consumption for the packet transmission and the large number of hops is considered [6]. For the networks that have energy as their limiting resource, the network lifetime related to the energy is one of the significant performance metrics [6]. The transport efficiency measures the bits per second per $\mathrm{Hz}$ per Joule of energy need to transport information reliably between source and destination node separated by end to end distance de which is greater than zero [6]. The maximum transport efficiency of the network can be maintained by decreasing the energy consumption for the network and also increasing the bandwidth efficiency of the network at the same time [6]. The relation between maximum transport efficiency of the network is linear function of end to end distance [9]. In [10] it is shown that with help of spatial ruse, we can increase bandwidth efficiency while suffering in the packet successful reception and with increase in number of hops there is decrease in the maximum transport efficiency regardless of the path loss exponents [11]. In this paper, we proposed a new routing protocol which is able to work in adverse condition where other protocols might shut down the working and may lead to network breakout. We termed this protocol as An Efficient on Demand Load Balancing Routing Protocol for MANETs (EDLBP). The protocol works on the technique termed as selective routing which is dynamic and is also able to allow SNCP i.e. Sub Network Control Protection for ad hoc and dynamic networks. 


\section{SYSTEM DESIGN AND MODEL}

The network model consists of $\mathrm{k}$ number of hops from source to destination. Therefore, the number of relaying nodes between source and destination will be $k-1$ [6][1]. Let $d_{e}$ be the end to end distance between source and the destination. If $\mathrm{d}_{\mathrm{i}}$ is the distance between the relaying nodes then the value of $\mathrm{d}_{\mathrm{i}}$ is given as: $\alpha_{\mathrm{i}} \mathrm{d}_{\mathrm{e}}$ where $0<\alpha_{\mathrm{i}}<1$. Note that for $\mathrm{k}$ number of hops the summation of $\alpha_{i} \geq 1$. This determines that it is not necessary that all the nodes are not always in the straight line [6]. The characteristics and the requirements of the nodes are: 1. Has a common power amplifier characteristics, (2) experiences the same propagation environment, (3) transmission is independent of each other that is from node to

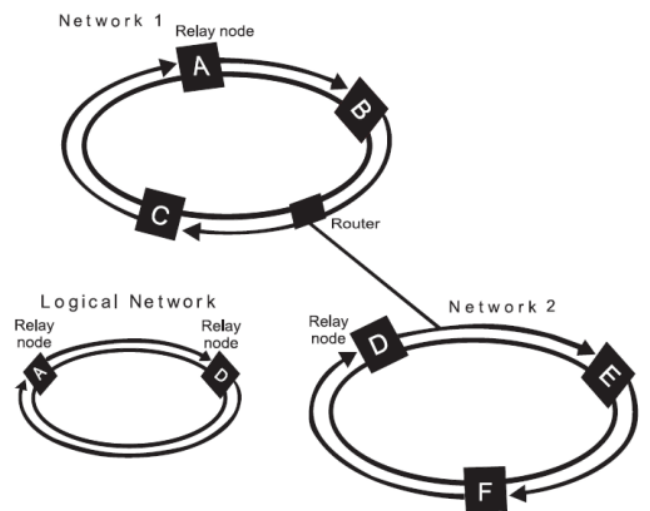

node, (4) requires energy $E_{p}[J]$ to process a received symbol.

\section{Figure 1}

The factors to be considered for the system model are $E_{p}$ as already defined is the receiver's processing energy, the power amplifier characteristics is described by two functions $f_{c}$ and $f_{o}$ [12]. As assumed in paper [12] $\mathrm{P}_{\text {in }}$ denote the input power to power amplifier, $\mathrm{P}_{\mathrm{dc}}$ the consumed power to drive the power amplifier to generate the desired output and $\mathrm{P}_{\text {out }}$ the desired output power of the power amplifier [6]. Now the characteristics can be given as:

$$
\begin{aligned}
& P_{\text {out }}=f_{o}\left(P_{\text {in }}\right) \\
& \left.P_{d c}=f_{c}\left(P_{\text {in }}\right) \ldots \ldots \ldots \ldots \ldots \ldots . . . . . . .6\right]
\end{aligned}
$$

Both the above function are strictly increasing function of $\mathrm{P}_{\text {in }}$ and the difference between the consumed power to drive the power amplifier and the desired output power of the power amplifier is equal to the heat loss in the power from the power amplifier of the transmitter on each node i.e. $\mathrm{P}_{\mathrm{h}}=\mathrm{P}_{\mathrm{dc}}$ $\mathrm{P}_{\text {out. }}$. Here $\mathrm{P}_{\mathrm{h}}$ is considered to be constant [6]. Also the simplifier power amplifier is considered with the following expressions:

$$
\begin{gathered}
\mathbf{f}_{\mathbf{o}}\left(\mathbf{P}_{\text {in }}\right)=\rho P_{\text {in }}, 0<P_{\text {in }}<P_{1} \\
P_{\text {SAT }}, P_{1}<P_{\text {in }} \leq P_{\text {max }} \\
f_{c}\left(P_{\text {in }}\right)=f_{o}\left(P_{\text {in }}\right)+P_{h} \ldots \ldots \ldots[6]
\end{gathered}
$$

where $\rho$ and $\mathrm{P}_{\mathrm{h}}$ are constants. Also it is considered that $\mathrm{P}_{\max }=$ $P_{1}$. The values for the constant are $\rho=50(17) \mathrm{dB}, \mathrm{P}_{1}=1.5 \mathrm{~mW}$, $\mathrm{P}_{\mathrm{SAT}}=75 \mathrm{~mW}$, and $\mathrm{P}_{\mathrm{h}}=35 \mathrm{~mW}$.

\section{LOAD BALANCING ROUTING PROTOCOL (EDLBP)}

An Efficient Load Balancing Routing Protocol is based upon two major factors one is the Selective routing and the other is the SNCP. This process is used to allow the controlled transmission to optimize the working of ad hoc Network.

\subsection{Selective Routing:}

The selective routing is based upon the amount of energy consumed for transmission of data by a node and the amount of energy left out for next session transmission. For this, we maintained a selection table along with the routing table is for routing. The packets transmitted in the network consume the energy in two formats one is the transmitter energy Tx and the second is the receiver processing energy Ep.

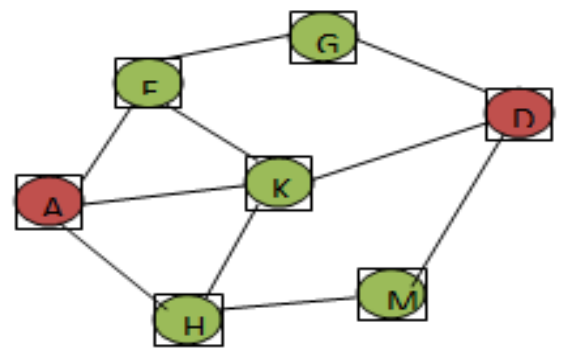

Figure 2

\begin{tabular}{|l|l|l|l|}
\hline $\begin{array}{l}\text { Head } \\
\text { Node }\end{array}$ & $\begin{array}{l}\text { Linked } \\
\text { Node }\end{array}$ & $\begin{array}{l}\text { Ep } \\
\text { (in } \\
\text { microns) }\end{array}$ & $\begin{array}{l}\text { Energy } \\
\text { Consumed } \\
\text { (Ec) }\end{array}$ \\
\hline $\mathrm{A}$ & $\mathrm{F}$ & 0.25 & 1.24 \\
\hline & $\mathrm{H}$ & 0.25 & 1.03 \\
\hline & $\mathrm{K}$ & 0.25 & 1.11 \\
\hline
\end{tabular}

Table1. Maintained For Each Node

The formulae used for calculation of the nodal energy are:

$$
\begin{aligned}
& \text { Ec }=\text { No. of packets transferred } * \text { Energy } \\
& \text { Required per Packet ................. (7) } \\
& \text { Energy Left= Total Energy }- \text { Ec ...... (8) }
\end{aligned}
$$

Energy table is maintained on the basis of calculation made using the above defined formulae. The path selection is made on basis of number of times the path is selected for transmission. Note that, energy consumed for transmission is directly proportional to number of times the path is chosen for transmission $(\mathrm{Nt})$ i.e.

Ec $\infty \mathbf{N t}$

Thus, more the path traversed more is the energy consumed and the more is the load on that path therefore, more is the danger of network failure.

$$
\operatorname{Load} \infty \mathbf{E c} \infty \mathrm{Nt} .
$$

Therefore, for load balancing the above defined parameters must be added to routing protocol for better and efficient transmission of data and more optimized network life. This 
selective routing can also be explained as algorithm defined as follows:

1. Traverse the network structure to maintain routing table.

2. Check acknowledgement to maintain energy table.

3. Start calculations from source towards destination.

4. If valid path transfers data update routing and energy table.

5. Maintain network connections live and check energy table if transmission halts.

6. In case of low energy, choose another optimized path.

7. On successful transmission, End transmission from source.

\subsection{Sub Network Control Protection:}

SNCP is the application of ring formation for delay management along with the load balancing. The ring formation allows the management of delays. This technique has been derived from the practical application of SNCP at various base station controllers of many telecommunication service providers. The technique has been verified by us using both simulations as well as the practical approach for the transmission process. The NAM animator results have been explained as follows:

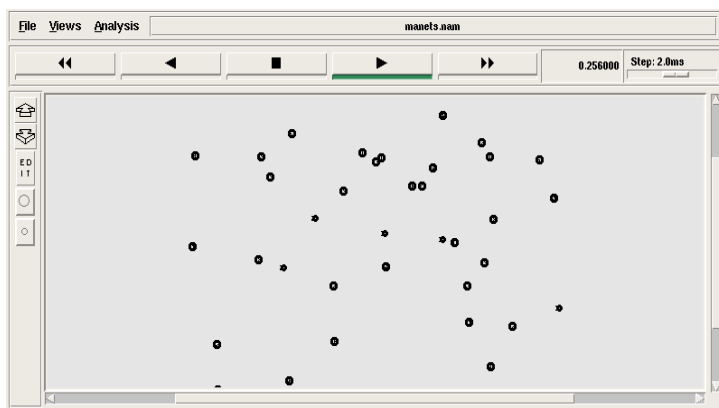

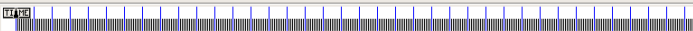

Figure 3

Figure 3 shows the initial structure of the nodes in network simulator.

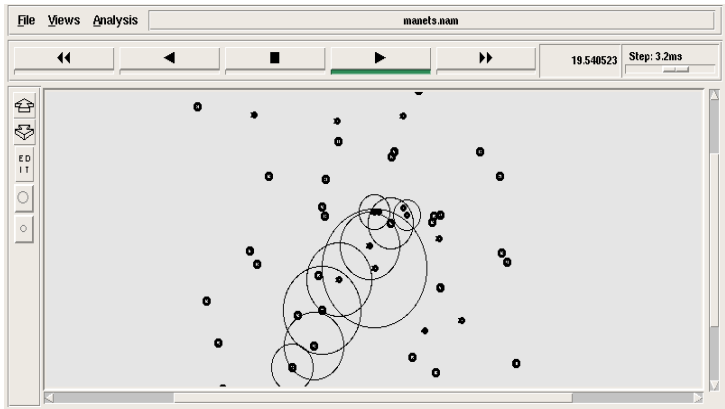

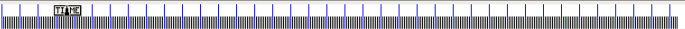

Figure 4 is the next phase that shows the transmission without use of our technique. It can be clearly noticed that number of active connections are much less as compared to the available connections.

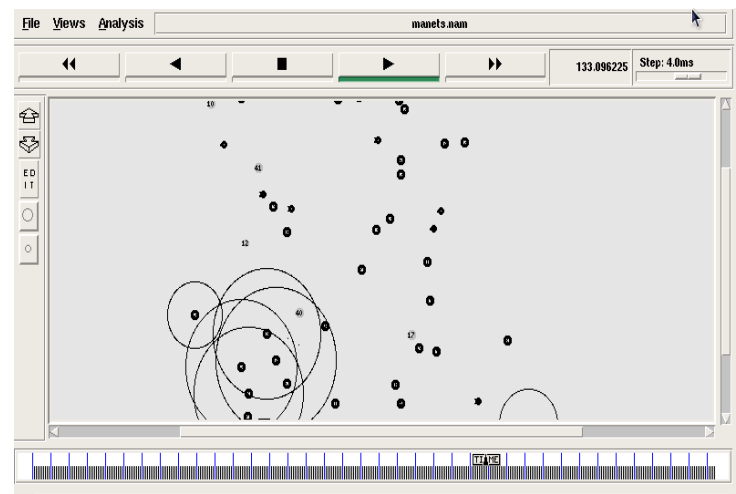

Figure 5

Figure 5 shows how the transmissions fails along with the time due to improper load balancing and due to increase in number of delays as the transmission proceeds towards its final stage.

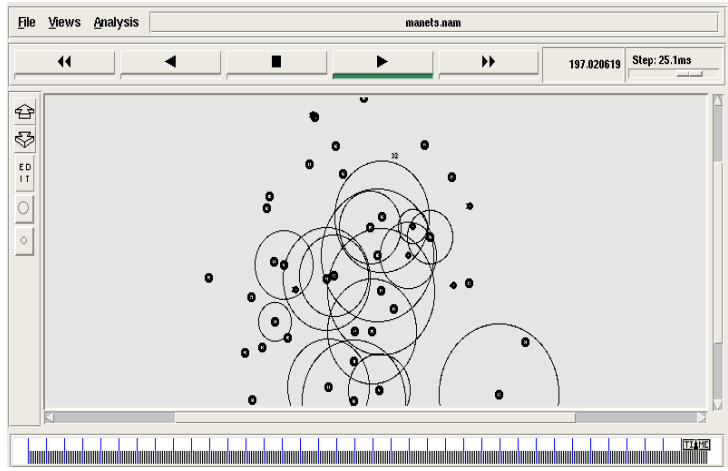

Figure 6

Figure 6 shows the application of our routing protocol that applies the selective routing as well as the SNCP technique. Clearly, there is more utilization of routing paths as well as of available number of connections. Also, this mode of transmission is free from any type delay arising due to propagation and transmission that may decrease the network life.

\subsection{Performance Metrics:}

We simulated the typical MANETs for hop and calculated the results for bandwidth, energy, throughput of the network structure taking mobility and the antenna range as the basis of the technique. We have taken values as constant to compute our results. The simulation has been performed for the area ranging over $1500 \times 1500$. Also, the values for the transmitter energy are taken to be $0.38 \mu \mathrm{J}$ and that of receiver processing energy is $0.25 \mu \mathrm{J}$. We concentrated on the following performance metrics: 
- Throughput: Throughput is the number of bits transferred during the transmission process. It is calculated I bits transferred per unit time. For our analysis the time considered is the simulation time.

- Mobility: Mobility is the new parameter that is highlighted in this paper. The readings have been taken by considering the scenario to be mobile and animations has also been recorded for the mobile nodes.

- End To End Delay: The delays are the latency caused in the process of transmission between the source and the destination. The delay considered in this paper is those that arise due to switching of the path to alternative route.

- Packet Lost/Received Ratio: The packet lost and received ratio is the number of packets arriving at destination correctly and this is taken into account to measure the performance of the network structure. More the packets received more is the life time of the network structure and more optimized is the path.

\section{SIMULATION RESULTS AND ANALYSIS}

The graphical analysis is carried out by comparing the trace file of the newly designed and previous version of protocol. The comparison is carried out by use of files present in the $\mathrm{x}$ graph of NS-2. The graphs taken by us are as follows:

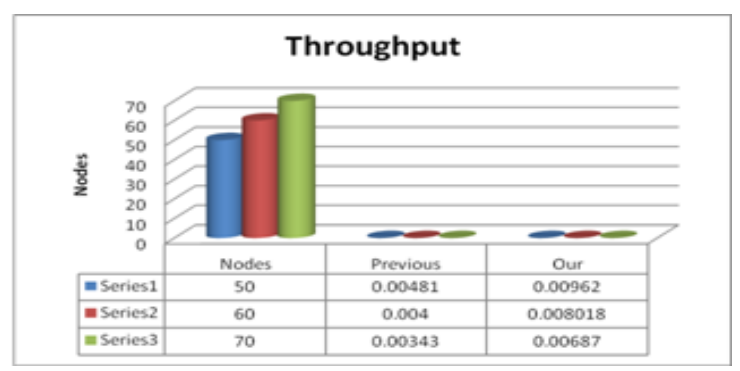

\section{Graph 1}

Graph 1 shows the result comparison of throughput for EDLBP and the AODV protocol. Clearly, as stated, EDLBP provides with more successful transmission of bit in particular time as compared to AODV protocol.

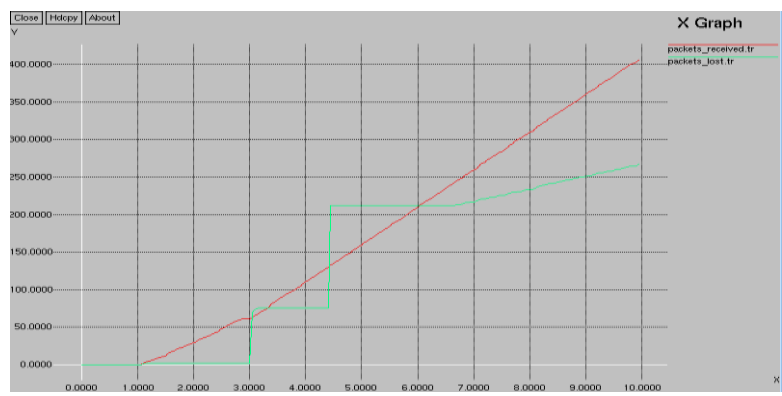

Graph 2
Graph 2 is the result of analysis between the packet lost and received for EDLBP. The result shows that more number of bits are transferred successfully as compared to AODV protocol.

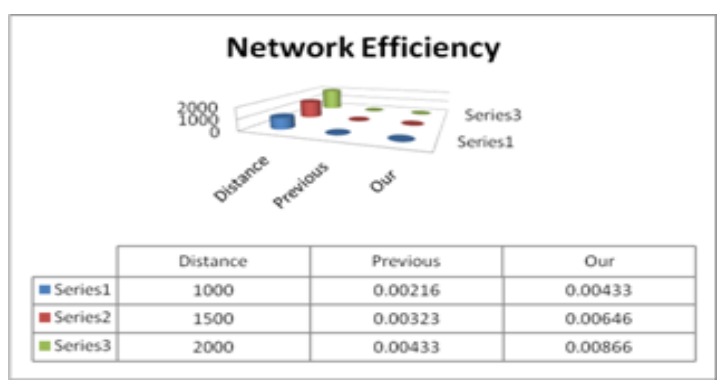

Graph 3

Graph 3 shows the result comparison of network efficiency for EDLBP and the AODV protocol. The network affiance for our protocol is improved by $50 \%$ approx.

\section{CONCLUSION}

Thus, from the paper, it is noticed that in order to increase the performance of the network, the load balancing and the delays of the system must be managed. In this paper, we have proposed EDLBP. As shown in the analysis carried out by us using both ns-2, we claim that the proposed model is capable of load balancing for network structure. Also the power consumption of the network has been minimized with increased efficiency there by resulting in increased lifetime of the network structure. Further, work can be carried out to reduce the number of retransmissions that will further optimize the network structure.

\section{REFERENCES}

[1] Dr. R.K. Singh, Vishal Sharma:'Dead State Recovery Based Power Optimization Routing Protocol for MANETs", HPAGC-2011, CCIS 169, pp.424-429,2011. (c) Springer-Verlag Berlin Heidelberg-2011.

[2] Sunil Taneja and Ashwani Kush:" A Survey of Routing Protocols in Mobile Ad Hoc abstract". International Journal of Innovation, Management and Technology, Vol. 1, No. 3, August 2010.

[3] M.Saravana karthikeyan, M.Murali, Dr.S.Sujatha:"Identifying performance metrics to maximize Manet's throughput"; 2010 International Conference on Advances in Computer Engineering.

[4] Manika Vinay Rali, Min Song, Sachin Shetty:"Virtual wired transmission scheme using directional antennas to improve energy efficiency in Wireless Mobile Ad Hoc Network"; 978-1-4244-2677-5, IEEE 2008.

[5] Computational Science @ the University of Oregon : http://www. csi.uoregon.edu. 
[6] Sehoon Kim, Jinkyu Lee and Ikjun Yeom," Modeling and Performance Analysis of Address Allocation Schemes for Wireless sensor networks", IEEE transactions on vehicular technology, vol. 57, NO. 1, JANUARY 2008.

[7] Rekha Patil, Dr. A. Damodaram:"'ost basedd power aware cross layer routing protocol for Manet"; 2008 IJCSNS.

[8] Changchun Bae and Wayne E. Stark:"A Tradeoff between Energy and Bandwidth Efficiency in Wireless Networks"; 2007 IEEE.
[9] V. Rodoplu and T. H. Meng: "Bits-per-Joule capacity of energy-limited wireless networks," IEEE Transaction Wireless Communications, vol.6(3), pp.857-865, March 2007.

[10] B. Rankov and A. Wittneben: "Spectral efficient protocols for half-duplex fading relay channels," IEEE Journal on Selected Areas in Communications, vol. 25, pp.379-389: Feb. 2007.

[11] Network simulator-2 www.isi.edu/nanam/ns/. 